\title{
Estudio computacional de las relaciones evolutivas de los receptores ionotrópicos NMDA, AMPA y kainato en cuatro especies de primates
}

\author{
Francy Johanna Moreno-Pedraza ${ }^{1}$, Leonardo Rene Lareo ${ }^{1 \dagger}$, Edgar Antonio Reyes-Montaño ${ }^{2}$ \\ ${ }^{1}$ Grupo de Bioquímica Molecular Computacional y Bioinformática, Departamento de Bioquímica, Facultad de Ciencias. Pontificia \\ Universidad Javeriana, Bogotá, D.C. Colombia. \\ ${ }^{2}$ Grupo de Investigación en Proteínas (GRIP) Departamento de Química, Universidad Nacional de Colombia, Ciudad Universitaria, \\ Edificio 451, Bogotá, Colombia. \\ *eareyesm@unal.edu.co
}

Recibido: 19-07-2010; Aceptado: 07-09-2010

\begin{abstract}
Resumen
Objetivo. Identificar la influencia de los cambios respecto a la estructura secundaria y a la relación evolutiva de los receptores NMDA, AMPA Y KAINATO en las especies Homo sapiens, Pan troglodytes, Pongo pygmaeus, y Macaca mulata. Materiales y métodos. Se recopilaron 91 secuencias correspondientes a los receptores NMDA, AMPA y Kainato y se sometieron a los programas de predicción de estructura secundaria, sitios de fosforilación, alineamientos múltiples, selección del modelo de evolución y predicción filogenética. Resultados. Se encontró que las subunidades GLUR5, NR2A, NR2C y NR3A presentaron cambios de estructura en la región C-terminal y aparición o pérdida de sitios de fosforilación en esta zona. Adicionalmente la predicción filogenética nos propone que las subunidades NR2 de NMDA son las más cercanas al nodo ancestral que da origen a los demás subunidades. Conclusiones. Los cambios de estructura y sitios de fosforilación en las subunidades GLUR5, NR2A, NR2C y NR3A nos sugieren variaciones en la interacción de la región C-terminal con proteínas quinasas y con proteínas con dominios PDZ lo cual podría afectar el tráfico y anclaje de las subunidades. Por otra parte la predicción filogenética nos propone que los cambios que se presentaron en las subunidades NR2 dieron origen a las demás subunidades de los receptores ionotrópicos de glutamato, básicamente porque son las subunidades de NMDA y en particular NR2D las que se encuentran más estrechamente relacionadas con el nodo ancestral que posiblemente dio origen a los iGluRs.
\end{abstract}

Palabras clave: receptores ionotrópicos de glutamato, iGluRs, NMDA, NR1, NR2A NR2C, NR3A, AMPA, GluR5.

\begin{abstract}
Computational study of the evolutionary relationships of the ionotropic receptors NMDA, AMPA and kainate in four species of primates. Objective. To identify the influence of changes on the secondary structure and evolutionary relationship of NMDA, AMPA and kainate receptors in Homo sapiens, Pan troglodytes, Pongo pygmaeus and Macaca mulatta. Materials and methods. We identified 91 sequences for NMDA, AMPA and kainate receptors and analyzed with software for predicting secondary structure, phosphorylation sites, multiple alignments, selection of protein evolution models and phylogenetic prediction. Results. We found that subunits GLUR5, NR2A, NR2C and NR3A showed structural changes in the C-terminal region and formation or loss of phosphorylation sites in this zone. Additionally the phylogenetic prediction suggests that the NMDA NR2 subunits are the closest to the ancestral node that gives rise to the other subunits. Conclusions. Changes in structure and phosphorylation sites in GLUR5, NR2A, NR2C and NR3A subunits suggest variations in the interaction of the C-terminal region with kinase proteins and with proteins with PDZ domains, which could affect the trafficking and anchoring of the subunits. On the other hand, the phylogenetic prediction suggests that the changes that occurred in the NR2 subunits gave rise to the other subunits of glutamate ionotropic receptors, primarily because the NMDA and particularly the NR2D subunits are the most closely related to the ancestral node that possibly gave rise to the iGluRs.
\end{abstract}

Key words: glutamate ionotropic receptors, iGluRs, NMDA, NR1, NR2A, NR2C, NR3A, AMPA, GluR5. 


\begin{abstract}
Resumo
Estudo computacional das relações evolutivas dos receptores ionotrópicos NMDA, AMPA e cainato em quatro espécies de primatas. Objetivo. Identificar a influência das mudanças da estrutura secundária e da relação evolutiva dos receptores NMDA, AMPA e cainato nas espécies Homo sapiens, Pan troglodytes, Pongo pygmaeus e Macaca mulatta. Materiais e métodos. Foram recopiladas 91 seqüências correspondentes aos receptores NMDA, AMPA e cainato e foram submetidos a programas de predição de estrutura secundária, sítios de fosforilação, alinhamentos múltiplos, seleção do modelo de evolução e predição da filogenia. Resultados. Descobrimos que as subunidades GLUR5, NR2A, NR2C e NR3A apresentaram alterações estruturais na região C-terminal e aparecimento ou perda de sítios de fosforilação nesta área. Além disso, a predição filogenética sugere ainda que as subunidades NR2 de NMDA são as mais próximas ao nó ancestral que dá origem às demais subunidades. Conclusões. As mudanças na estrutura e nos sítios de fosforilação nas subunidades GLUR5, NR2A, NR2C e NR3A sugerem variações na interação da região C-terminal com proteínas quinase e com proteínas de domínios PDZ que poderia afetar o tráfego e fixação das subunidades. Além disso, a predição filogenética sugere que as mudanças ocorridas nas subunidades NR2 deram origem às outras subunidades de receptores ionotrópicos de glutamato, principalmente porque são subunidade NMDA e particularmente NR2D aquelas que são mais estreitamente relacionadas com o nó ancestral que provavelmente deu origem aos iGluRs.
\end{abstract}

Palavras-chave: receptores ionotrópicos de glutamato, iGluRs, NMDA, NR1, NR2A NR2C, NR3A, AMPA, GluR5.

\section{Introducción}

Una forma de entender la evolución humana es a través del estudio de los primates básicamente por similitudes morfológicas, bioquímicas y de comportamiento en respuesta a condiciones ambientales parecidas; por ejemplo una característica importante es que los primates presentan cerebros con mayor tamaño respecto al tamaño corporal en relación a otras especies (1). Esta tendencia es mayor en los seres humanos y es una característica evolutiva que favorece los procesos de memoria y aprendizaje (2). Adicionalmente las habilidades cognitivas están directamente relacionadas con el incremento en el tamaño del cerebro (3).

Varios de los aportes que hasta el momento se han realizado respecto al funcionamiento y estructura del cerebro, del sistema nervioso central (SNC), de los procesos sinápticos, entre otros, para los mamíferos y en especial para el ser humano involucran al glutamato. Este aminoácido excitatorio es uno de los principales neurotransmisores del SNC, el cual actúa a través de receptores ionotrópicos y metabotrópicos (4).

Los receptores ionotrópicos (iGLURs) conforman canales permeables a iones como $\mathrm{Na}^{+}, \mathrm{Ca}^{2+}$ y $\mathrm{K}^{+}$; se dividen en cuatro grupos denominados NMDA, AMPA, Kainato y Delta. Los receptores metabotrópicos (mGLURs) están acoplados a segundos mensajeros como diacilglicerol, inositol-3-fosfato y AMP cíclico $(4,5)$.

Los iGLURs son mediadores importantes de las señales de excitación sináptica, y contribuyen a los procesos de memoria y aprendizaje (6), así como en diversas neuropatologías como la epilepsia y la isquemia. Su función principal es recibir la señal química del glutamato que se une a un dominio extracelular del receptor, generando la permeabilidad del mismo a cationes de $\mathrm{Na}^{+}$, $\mathrm{Ca}^{2+} \mathrm{y} \mathrm{K}^{+}$que contribuyen a una variación en el potencial eléctrico de la membrana post-sináptica (7).

Molecularmente los iGLURs se caracterizan por ser proteínas integrales de membrana que presentan tres dominios transmembranales designados como M1, M3 y M4, un bucle reentrante en la membrana en la zona citoplasmática designado como M2, el extremo aminoterminal localizado en la zona extracelular y el carboxiterminal en la zona citoplasmática, dos dominios de unión a ligando designados como S1 y S2 que se encuentran adyacentes al dominio M1 y en el bucle que se forma entre los dominios M3 y M4 y un dominio flip/flop representado por una estructura helicoidal, en el lado opuesto del dominio de unión a ligando S2 (8).

En el aspecto evolutivo, una predicción filogenética relacionó la familia de genes iGLURs en animales y los genes GLR (glutamate receptor-like) en plantas como Arabidopsis thaliana, los análisis por parsimonia demostraron que los genes GLR son el grupo basal para la familia de receptores de glutamato, esta ubicación indica que la divergencia entre los receptores putativos de la planta y los iGLURs en animales surgió antes de la divergencia entre los iGLURs en animales (NMDA, AMPA, Kainato). A partir de estos resultados se sugiere que la señalización célula-célula por aminoácidos excitatorios en el cerebro de los animales, surgió de un mecanismo de señalización primitivo que existió inicialmente en las plantas (9).

A través, de procedimientos in silico se analizó la evolución molecular del dominio C-terminal en el receptor 
NMDA, los resultados demuestran que la evolución generó un dominio C-terminal no estructurado en la subunidad NR2 en el linaje de los vertebrados, el cual participa en complejos de señalización que influyen en la plasticidad neuronal y puede contribuir a la diferenciación de las especies en relación a su comportamiento y función cognitiva, teniendo en cuenta que esta región es mucho más corta en especies invertebradas y sólo presenta el dominio de señalización denominado PDZ (10).

En este trabajo, se relacionaron evolutivamente las especies Homo sapiens, Pan troglodytes, Pongo pygmaeus y Macaca mulata, a través de las secuencias de los receptores ionotrópicos NMDA, AMPA y KAINATO, empleando herramientas computacionales que permitieron: identificar los cambios que se presentaron en los receptores, reconocer la influencia de estos cambios en la estructura secundaria y proponer una relación evolutiva a partir de las inferencias filogenéticas para algunos de estos receptores.

\section{Materiales y métodos}

\section{Recopilación de datos}

Las secuencias proteicas de las subunidades de los receptores ionotrópicos tipo AMPA (GLUR 1, GLUR 2, GLUR 3 y GLUR 4), NMDA (NR 1, NR2A, NR2B, NR2C, NR2D, NR3A y NR3B) y Kainato (GLUR 5, GLUR 6, GLUR 7, KA 1 y KA 2) en las especies Homo sapiens, Pan troglodytes, Pongo pygmaeus, Macaca mulata y Rattus norvegicus se obtuvieron de las bases de datos NCBI (http://www.ncbi. nlm.nih.gov/sites/entrez?db=Protein\&itool=toolbar) y Ensembl (http://www.ensembl.org/index.html). Las secuencias de la especie Rattus norvegicus se incluyeron en el estudio como representación del grupo externo, el cual es importante para las reconstrucciones filogenéticas porque permite reconocer en qué parte de los árboles se sitúa el nodo ancestral.

\section{Alineamientos múltiples}

Todas las secuencias se sometieron al programa de alineamientos múltiples ClustalX (11), empleando los siguientes parámetros: gap opening 15 , gap extension 0,3 y Gonnet como matriz interna. Se realizaron cuatro tipos de alineamientos múltiples por subunidad (incluye las secuencias de las cinco especies por subunidad de receptor), por receptor (incluye las secuencias de las cinco especies en las subunidades que representan el receptor), por especie (incluye todas las subunidades para los tres receptores en una especie) y uno con todas las secuencias para los tres receptores en las cinco especies.

\section{Predicción de estructura secundaria}

Las secuencias de las subunidades en las que se identificaron una mayor proporción de cambios en la región C-terminal fueron sometidas al programa de predicción de estructura secundaria Psipred Protein Structure Prediction Server (http://www.psipred.net/psiform.html), este servidor incorpora redes neuronales para reconocer patrones de estructura secundaria a partir de una base de datos de proteínas resueltas estructuralmente en estudios experimentales (12).

\section{Predicción de sitios de fosforilación}

Las secuencias de las subunidades en las que se identificaron varios cambios en la región C-terminal fueron sometidas al programa de predicción de sitios de fosforilación en proteínas eucariotas, NetPhos 2.0 (http://www.cbs.dtu.dk/ services/NetPhos/), el cual trabaja con métodos de redes neuronales artificiales, tomando como base secuencias verificadas experimentalmente como sitios de fosforilación, estas redes reconocen patrones de secuencias biológicas de 7-12 residuos de aminoácidos que pueden ser reconocidas por quinasas y la especificidad en el reconocimiento está determinada por las características de hidropatía de los residuos aminoacídicos adyacentes al residuo fosforilado (13).

\section{Selección del modelo de evolución}

Los alineamientos también fueron sometidos al programa de selección de modelos evolutivos para proteínas Prottest (http://darwin.uvigo.es/software/prottest_server.html) (14), dado que los modelos evolutivos son la base de las reconstrucciones filogenéticas porque emplean matrices que describen la probabilidad de cambio de un aminoácido por otro. Para este procedimiento fue necesario descargar el programa Prottest con el propósito de mejorar la eficiencia computacional, el programa arrojó los resultados para los alineamientos por subunidad, pero no fue posible obtenerlos para los demás alineamientos debido a que por el tamaño de los archivos se requería de una mayor plataforma computacional, por lo tanto fue necesario emplear el servidor PALM (http://palm.iis.sinica.edu.tw/index.html) el cual está representado por un marco integrado de programas como ClustalW, PhyML, ModelTest, ProtTest, y varios programas internos, evalúa la aptitud de 112 modelos de 
sustitución de secuencias de proteínas con las puntuaciones en los diversos criterios y realiza la estimación filogenética por máxima verosimilitud (15), que resultó importante como resultados preliminares en las inferencias filogenéticas.

\section{Estimación filogenética}

Una vez se determinó cual fue el mejor modelo de evolución para los diferentes alineamientos se realizó la predicción filogenética empleando el programa PHYML (16), que emplea el método de reconstrucción filogenética por de máxima verosimilitud. La base de este método es un algoritmo que inicia desde un árbol inicial construido por un método rápido basado en las distancias y modifica este árbol para mejorar sus probabilidades en cada iteración. Debido a este ajuste simultáneo de la topología y longitud de las ramas, sólo unas pocas iteraciones son suficientes para llegar a un punto de topología óptimo (16). Como prueba de confiabilidad se realizó un Bootstrap de 100 que es un valor estándar que emplean los servidores cuando se aplica esta prueba, fue necesario hacer uso de varios servidores que trabajaran con el programa Phyml, debido a que se presentaron limitantes en la disponibilidad de los modelos de evolución los cuales se encuentran reportados en los recursos electrónicos.

\section{Resultados}

\section{Recopilación de datos y Alineamientos múltiples}

Los códigos correspondientes a las secuencias de las proteínas que se emplearon en este estudio se reportan en la tabla 1, La búsqueda se inició en el servidor NCBI empleando los posibles nombres que se pueden asignar a las subunidades con base en los reportes literarios, identificar si existían reportes en las especies seleccionadas, y solo seleccionar aquellas secuencias con longitudes muy cercanas a las reportadas en estudios de tipo experimental, para evitar seleccionar secuencias que correspondieran a duplicaciones del gen o fragmentos de este. Los resultados encontrados fueron limitados, no se encontraron reportes para algunas de las especies seleccionadas y fue necesario complementar la búsqueda con la base de datos Ensembl, en este caso la búsqueda se hizo a partir de los nombres de

Tabla 1. Códigos de acceso de las secuencias empleadas en el estudio

\begin{tabular}{|c|c|c|c|c|c|}
\hline \multirow{3}{*}{$\begin{array}{c}\text { NOMBRE } \\
\text { DEL } \\
\text { RECEPTOR }\end{array}$} & \multicolumn{5}{|c|}{ ESPECIES } \\
\hline & Homo sapiens & Pongo pygmaeus & Pan troglodytes & Macaca mulata & Rattus norvegicus \\
\hline & & & AMPA & & \\
\hline GluR1 & NP_000818 & ENSPPYP00000017871 & XP_001169416 & XP_001111040 & NP_113796 \\
\hline GluR 2 & NP_000817 & ENSPPYP00000016933 & XP_001141404 & XP_001095129 & NP_058957 \\
\hline GluR 3 & NP_015564 & $x$ & ENSPTRP00000038297 & ENSMMUP00000011880 & NP_116785 \\
\hline GluR 4 & NP_000820 & $\mathrm{x}$ & ENSPTRP00000007287 & ENSMMUP00000014398 & NP_058959 \\
\hline & & & NMDA & & \\
\hline $1-1 a$ & ENSP00000360614 & $\mathrm{X}$ & ENSPTRP00000036984 & ENSMMUP00000002231 & AAB50926 \\
\hline $1-1 b$ & ENSP00000360608 & ENSPPYP00000022212 & ENSPTRP00000041565 & ENSMMUP00000040250 & AAB50928 \\
\hline $1-2 b$ & ENSP00000360605 & $x$ & ENSPTRP00000036983 & ENSMMUP00000002232 & AAB50929 \\
\hline $1-3 b$ & ENSP00000360616 & ENSPPYP00000022211 & ENSPTRP00000036985 & ENSMMUP00000040251 & AAB50931 \\
\hline $1-4 b$ & ENSP00000360615 & $x$ & ENSPTRP00000036982 & ENSMMUP00000002228 & AAB50933 \\
\hline NR2A & NP_000824 & ENSPPYP00000008016 & NP_001029361 & ENSMMUP00000013265 & NP_036705 \\
\hline NR2B & NP_000825 & ENSPPYP00000004930 & XP_528744 & ENSMMUP00000025129 & NP_036706 \\
\hline $\mathrm{NR} 2 \mathrm{C}$ & NP_000826 & ENSPPYP00000009666 & XP_511665 & ENSMMUP00000007584 & NP_036707 \\
\hline NR2D & NP_000827 & ENSPPYP00000011404 & $x$ & ENSMMUP00000007863 & NP_073634 \\
\hline NR3A & NP_597702 & ENSPPYP00000021802 & ENSPTRP00000039833 & ENSMMUP00000021930 & ENSRNOP00000007957 \\
\hline NR3B & NP_619635 & $x$ & $\begin{array}{l}\text { ENSPTRP00000049173 } \\
\text { KAINATO }\end{array}$ & $x$ & NP_579842 \\
\hline GluR 5 & NP_000821 & ENSPPYP00000012647 & XP_001161838 & ENSMMUP00000006495 & NP_058937 \\
\hline GluR 6 & NP_068775 & ENSPPYP00000018887 & ENSPTRP00000031512 & $x$ & NP_062182 \\
\hline GluR 7 & NP_000822 & ENSPPYP00000001771 & ENSPTRP00000000961 & ENSMMUP00000012661 & NP_852038 \\
\hline KA1 & NP_055434 & ENSPPYP00000004555 & NP_001029331 & ENSMMUP00000029417 & NP_036704 \\
\hline KA2 & NP_002079 & ENSPPYP00000011230 & $X P \_524469$ & ENSMMUP00000022947 & NP_113696 \\
\hline
\end{tabular}


los genes, los resultados mostraron los transcriptos para cada gen y las proteínas que codifican, para seleccionar las secuencias de aminoácidos se tuvieron en cuenta los criterios mencionados anteriormente, finalmente se recopilaron 91 secuencias de 100 posibles con la limitante de 5 secuencias en la especie Pongo pygmaeus, una secuencia en la especie Pan troglodytes, y 2 secuencias en la especie Macaca mulata.
Los resultados de los alineamientos múltiples por subunidad para cada receptor permitieron identificar los cambios que se presentan en cada secuencia proteica, las subunidades que se destacaron por presentar una mayor proporción de sustituciones fueron GluR5, NR2A, NR2C y NR3A (Tablas 2, 3), para cada columna de cambio, el primer aminoácido representa el aminoácido que se mantiene conservado en las demás especies de estudio y el

Tabla 2. Cambios Identificados en las Subunidades GLUR5

\begin{tabular}{|c|c|c|c|c|c|c|c|c|c|c|c|c|c|c|c|c|c|}
\hline \multirow{2}{*}{$\begin{array}{l}\text { Receptor } \\
\text { GLUR5 }\end{array}$} & \multirow{2}{*}{$\begin{array}{c}\text { Especie } \\
\text { Homo sapiens }\end{array}$} & \multirow{2}{*}{$\begin{array}{c}\text { Posición } \\
838\end{array}$} & \multicolumn{3}{|c|}{ Cambio } & \multirow{2}{*}{$\begin{array}{c}\text { Posición } \\
839\end{array}$} & \multicolumn{3}{|c|}{ Cambio } & \multirow{2}{*}{$\begin{array}{c}\text { Posición } \\
840\end{array}$} & \multicolumn{3}{|c|}{ Cambio } & \multirow{2}{*}{$\begin{array}{c}\text { Posición } \\
841\end{array}$} & \multicolumn{3}{|c|}{ Cambio } \\
\hline & & & $M$ & por & C & & $E$ & por & $F$ & & $\bar{E}$ & por & $\bar{F}$ & & $\mathrm{~L}$ & por & $Y$ \\
\hline & & 843 & 1 & por & $\mathrm{L}$ & 844 & $\mathrm{~S}$ & por & $Q$ & 845 & L & por & c & 847 & $\mathrm{~N}$ & por & Q \\
\hline & & 848 & $Q$ & por & $T$ & 849 & $\mathrm{~K}$ & por & $\mathrm{H}$ & 850 & K & por & $P$ & 851 & L & por & $\mathrm{T}$ \\
\hline & & 852 & K & por & $N$ & 853 & K & por & $\mathrm{S}$ & 854 & $K$ & por & $T$ & 856 & $R$ & por & G \\
\hline & & 858 & $\mathrm{~K}$ & por & $T$ & 859 & G & por & L & 860 & $\mathrm{~K}$ & por & $S$ & 861 & $\mathrm{~S}$ & por & $\mathrm{T}$ \\
\hline & & 862 & $\mathrm{~S}$ & por & D & 863 & $\mathrm{~F}$ & por & $\mathrm{L}$ & 864 & $T$ & por & $E$ & 865 & $\mathrm{~S}$ & por & C \\
\hline & & 866 & 1 & por & G & 867 & L. & por & K & 868 & $T$ & por & L & 869 & C & por & 1 \\
\hline & & 875 & $Q$ & por & 1 & 878 & $E$ & por & $Q$ & 879 & $T$ & por & $\mathrm{S}$ & 880 & V & por & $\mathrm{S}$ \\
\hline & Pan troglodytes & 838 & M & por & C & 839 & $E$ & por & $\mathrm{F}$ & 840 & $E$ & por & $\mathrm{F}$ & 841 & L & por & Y \\
\hline & & 843 & 1 & por & $\mathrm{L}$ & 844 & $\mathrm{~S}$ & por & $Q$ & 845 & $L$ & por & C & 847 & $\mathrm{~N}$ & por & Q \\
\hline & & 848 & $Q$ & por & $T$ & 849 & $\mathrm{~K}$ & por & $\mathrm{H}$ & 850 & K & por & $P$ & 851 & L & por & $\mathrm{T}$ \\
\hline & & 852 & $\mathrm{~K}$ & por & $\mathrm{N}$ & 853 & K & por & $\mathrm{S}$ & 854 & $\mathrm{~K}$ & por & $T$ & 856 & R & por & G \\
\hline & & 858 & $\mathrm{~K}$ & por & $\mathrm{T}$ & 859 & G & por & L & 860 & $\mathrm{~K}$ & por & $\mathrm{S}$ & 861 & $\mathrm{~S}$ & por & $\mathrm{T}$ \\
\hline & & 862 & $\mathrm{~S}$ & por & D & 863 & $\mathrm{~F}$ & por & $\mathrm{L}$ & 864 & $\mathrm{~T}$ & por & E & 865 & $\mathrm{~S}$ & por & C \\
\hline & & 875 & $Q$ & por & 1 & 878 & $E$ & por & $Q$ & 879 & $T$ & por & $\mathrm{S}$ & 880 & V & por & $\mathrm{S}$ \\
\hline & Pongo pygmaeus & 296 & $\mathrm{H}$ & por & V & & & & & & & & & & & & \\
\hline & Rattus norvegicus & 108 & $\mathrm{~N}$ & por & S & 109 & $\mathrm{~K}$ & por & $\mathrm{K}$ & 127 & 1 & por & V & 173 & S & por & $\mathrm{P}$ \\
\hline & & 174 & G & por & A & 188 & G & por & G & 341 & $\mathrm{H}$ & por & $\mathrm{R}$ & 348 & $\mathrm{~N}$ & por & $\mathrm{D}$ \\
\hline & & 398 & $\mathrm{~S}$ & por & G & 400 & K & por & K & 402 & $\mathrm{~K}$ & por & $\mathrm{R}$ & 404 & $\mathrm{~S}$ & por & $\mathrm{N}$ \\
\hline & & 459 & 1 & por & $\mathrm{L}$ & 696 & $\mathrm{~T}$ & por & $\mathrm{S}$ & 700 & $\mathrm{R}$ & por & $\mathrm{K}$ & 790 & $\mathrm{~N}$ & por & $\mathrm{S}$ \\
\hline & & 824 & 1 & por & $\mathrm{L}$ & 833 & 1 & por & $\mathrm{V}$ & & & & & & & & \\
\hline
\end{tabular}

Los cambios sombreados en verde representan cambios no conservados, los sombreados en amarillo son cambios menos conservados, los no sombreados son cambios conservados y los que se resaltan con un recuadro negro son cambios que se ubican en los dominios de unión a ligando S1 y/o S2.

Tabla 3. Cambios Identificados en las Subunidades NR2A.

\begin{tabular}{|c|c|c|c|c|c|c|c|c|c|c|c|c|c|c|c|c|c|}
\hline \multirow{2}{*}{$\begin{array}{c}\text { Receptor } \\
\text { NR2A }\end{array}$} & \multirow{2}{*}{$\begin{array}{c}\text { Especie } \\
\text { Homo sapiens }\end{array}$} & \multirow{2}{*}{$\begin{array}{c}\text { Posición } \\
354\end{array}$} & \multicolumn{3}{|c|}{ Cambio } & \multicolumn{2}{|l|}{ Posición } & \multicolumn{2}{|c|}{ Cambio } & \multirow{2}{*}{$\begin{array}{c}\text { Posición } \\
991\end{array}$} & \multicolumn{3}{|c|}{ Cambio } & \multirow{2}{*}{$\begin{array}{c}\text { Posición } \\
1010\end{array}$} & \multicolumn{3}{|c|}{ Cambio } \\
\hline & & & $Q$ & por & $\mathrm{H}$ & 879 & $\mathrm{~N}$ & por & $\mathrm{S}$ & & $\mathrm{H}$ & por & $\mathrm{V}$ & & G & por & A \\
\hline & & 1127 & $\bar{H}$ & por & P & 1194 & $\mathrm{~L}$ & por & $M$ & & & & & & & & \\
\hline & \multirow{2}{*}{ Pan troglodytes } & 109 & $A$ & por & $\mathrm{N}$ & 354 & $Q$ & por & $\mathrm{H}$ & 991 & $\mathrm{H}$ & por & V & 1010 & G & por & A \\
\hline & & 1053 & $\mathrm{H}$ & por & $\mathrm{P}$ & 1127 & $\mathrm{H}$ & por & P & 1131 & $\mathrm{~F}$ & por & $\mathrm{L}$ & 1146 & $\mathrm{H}$ & por & Q \\
\hline & \multirow{5}{*}{$\begin{array}{l}\text { Pongo pygmaeus } \\
\text { Macaca mulata }\end{array}$} & 174 & $Q$ & por & $\mathrm{K}$ & 1010 & A & por & G & 1063 & V & por & G & 1151 & L & por & $P$ \\
\hline & & 4 & $P$ & por & $\mathrm{S}$ & 19 & V & por & $M$ & 92 & $\mathrm{H}$ & por & Q & 95 & V & por & I \\
\hline & & 155 & $E$ & por & D & 302 & v & por & I & 914 & $\mathrm{~L}$ & por & $\hat{M}$ & 944 & Q & por & $\mathrm{H}$ \\
\hline & & 1010 & A & por & G & 1034 & i & por & V & 1143 & $\mathrm{~N}$ & por & $\mathrm{K}$ & 1152 & $\mathrm{~S}$ & por & $P$ \\
\hline & & 1153 & $\mathrm{~N}$ & por & D & 1154 & $\mathrm{~N}$ & por & $\mathrm{T}$ & 1155 & D & por & $\mathrm{E}$ & 1236 & $G$ & por & R \\
\hline & \multirow[t]{13}{*}{ Rattus norvegicus } & 12 & M & por & $\mathrm{L}$ & 26 & 1 & por & $\mathrm{N}$ & 34 & A & por & $T$ & 92 & $\mathrm{H}$ & por & $Q$ \\
\hline & & 95 & V & por & 1 & 155 & $E$ & por & D & 160 & V & por & 1 & 297 & $M$ & por & A \\
\hline & & 299 & $\mathrm{R}$ & por & $\mathrm{K}$ & 302 & V & por & $\mathrm{T}$ & 304 & $M$ & por & $\mathrm{L}$ & 309 & $\mathrm{P}$ & por & Q \\
\hline & & 680 & $\mathrm{~K}$ & por & $\mathrm{R}$ & 684 & $\mathrm{~K}$ & por & $\mathbf{R}$ & 895 & A & por & $\mathrm{T}$ & 905 & $M$ & por & V \\
\hline & & 915 & $M$ & por & 1 & 926 & $\mathrm{E}$ & por & D & 944 & $Q$ & por & $\mathrm{H}$ & 949 & $\mathrm{~N}$ & por & S \\
\hline & & 979 & V & por & G & 992 & D & por & $E$ & 994 & 1 & por & $\mathrm{L}$ & 1000 & $\mathrm{~S}$ & por & $N$ \\
\hline & & 1010 & A & por & K & 1029 & $M$ & por & V & 1039 & $\mathrm{~N}$ & por & $\mathrm{S}$ & 1050 & $\mathrm{~S}$ & por & $\mathrm{N}$ \\
\hline & & 1063 & V & por & $M$ & 1073 & $E$ & por & D & 1075 & $E$ & por & D & 1079 & $\mathrm{~L}$ & por & $M$ \\
\hline & & 1083 & $S$ & por & A & 1100 & G & por & $S$ & 1112 & V & por & 1 & 1119 & D & por & G \\
\hline & & 1161 & $S$ & por & A & 1176 & $T$ & por & G & 1178 & $E$ & por & D & 1236 & $G$ & por & $\mathrm{R}$ \\
\hline & & 1238 & $Q$ & por & $E$ & 1245 & A & por & $\mathrm{S}$ & 1252 & $\bar{L}$ & por & $\mathrm{F}$ & 1260 & $\mathrm{~S}$ & por & $\mathrm{N}$ \\
\hline & & 1335 & $\mathrm{~T}$ & por & $\bar{A}$ & 1343 & $\mathrm{~S}$ & por & $T$ & 1344 & $\mathrm{H}$ & por & $Y$ & 1345 & R & por & G \\
\hline & & 1393 & $\mathrm{~N}$ & por & $\mathrm{S}$ & 1410 & $\mathrm{~N}$ & por & $T$ & & & & & & & & \\
\hline
\end{tabular}

Los cambios sombreados en verde representan cambios no conservados, los sombreados en amarillo son cambios menos conservados, los no sombreados son cambios conservados y los que se resaltan con un recuadro negro son cambios que se ubican en los dominios de unión a ligando S1 y/o S2. 
segundo es el aminoácido sustituyente para la especie en particular.

En la subunidad Glur5 el mayor número de sustituciones se presentaron en las especies Homo sapiens y Pan troglodytes, entre los aminoácidos 843-875 (Tabla 2), los cuales se distribuyeron hacia el extremo C-terminal, de igual forma ocurrió en la subunidad NR2A para las cinco especies, en NR2C los cambios se ubican en el N y Cterminal, la especie con mayor proporción de sustituciones es Macaca mulata y en ninguno de los casos se involucran los dominios de unión a ligando (Tabla 3, 4).
Se encontró que la posición 596 es muy variable en la subunidad NR3A y participa en el dominio de unión S1, en esta posición Homo sapiens y Pan troglodytes presentan una arginina, Macaca mulata y Rattus norvegicus presentan una serina y Pongo pygmaeus una asparagina (Tabla 5), adicionalmente Macaca mulata presenta un cambio conservado en la posición E804Q (dominio S2). La especie Rattus norvegicus como grupo externo se caracterizó por presentar un considerable número de sustituciones en comparación a las cuatro especies de primates esto se debe a su distancia evolutiva dado que pertenece a otro orden.

Tabla 4. Cambios Identificados en las Subunidades NR2C.

\begin{tabular}{|c|c|c|c|c|c|c|c|c|c|c|c|c|c|c|c|c|c|}
\hline & \multirow{2}{*}{$\begin{array}{c}\text { Especie } \\
\text { Homo sapiens }\end{array}$} & \multirow{2}{*}{$\begin{array}{c}\text { Posición } \\
18\end{array}$} & \multicolumn{3}{|c|}{ Cambio } & \multirow{2}{*}{$\begin{array}{c}\text { Posición } \\
27\end{array}$} & \multicolumn{2}{|c|}{ Cambio } & \multicolumn{2}{|c|}{ Posición } & \multicolumn{3}{|c|}{ Cambio } & \multirow{2}{*}{$\frac{\text { Posición }}{70}$} & \multicolumn{3}{|c|}{ Cambio } \\
\hline & & & G & por & $\mathrm{S}$ & & V & por & $\mathrm{F}$ & 52 & $\mathrm{~N}$ & por & $\mathrm{T}$ & & $\mathrm{R}$ & por & $\mathrm{H}$ \\
\hline \multirow{52}{*}{ NR2C } & & 81 & G & por & D & 300 & A & por & V & 577 & $\mathrm{~S}$ & por & A & 1108 & $T$ & por & I \\
\hline & & 1193 & C & por & R & & & & & & & & & & & & \\
\hline & Pan troglodytes & 13 & G & por & $\mathrm{S}$ & 22 & V & por & $\mathrm{F}$ & 67 & H & por & $\mathrm{R}$ & 295 & A & por & V \\
\hline & & 329 & L & por & Q & 341 & $\mathrm{R}$ & por & $P$ & 342 & $\mathrm{H}$ & por & $Q$ & 827 & $\mathrm{~s}$ & por & $T$ \\
\hline & & 862 & $\bar{E}$ & por & $\bar{S}$ & 864 & L & por & $Q$ & 866 & $H$ & por & $\bar{A}$ & 868 & A & por & $\mathrm{P}$ \\
\hline & & 869 & W & por & $\mathrm{S}$ & 895 & $T$ & por & 1 & 897 & $R$ & por & P & 903 & G & por & $\mathrm{S}$ \\
\hline & Pongo pygmaeus & 22 & $\mathrm{~F}$ & por & V & 47 & $\mathrm{~N}$ & por & $T$ & 282 & $\mathrm{H}$ & por & $\mathrm{Y}$ & 293 & V & por & A \\
\hline & & 353 & V & por & 1 & 824 & $\mathrm{~S}$ & por & $T$ & 1047 & $R$ & por & Q & 1118 & $Q$ & por & L \\
\hline & Macaca mulata & 27 & $\mathrm{~F}$ & por & V & 112 & $\mathrm{~T}$ & por & $\mathrm{s}$ & 115 & E & por & V & 116 & P & por & $\mathrm{H}$ \\
\hline & & 117 & G & por & V & 118 & $\mathrm{~S}$ & por & $Q$ & 119 & A & por & $\mathrm{T}$ & 120 & $\mathrm{~F}$ & por & $\mathrm{H}$ \\
\hline & & 121 & $\mathrm{~L}$ & por & V & 122 & Q & por & $\mathrm{P}$ & 123 & L & por & $\mathrm{s}$ & 124 & G & por & C \\
\hline & & 125 & V & por & $\mathrm{L}$ & 126 & S & por & R & 127 & L & por & P & 128 & $E$ & por & G \\
\hline & & 129 & Q & por & $\bar{T}$ & 130 & Q & por & $\mathrm{R}$ & 132 & $Q$ & por & G & 133 & $\bar{v}$ & por & $R$ \\
\hline & & 134 & L & por & A & 135 & $\bar{F}$ & por & $\mathrm{V}$ & 136 & $\bar{K}$ & por & L & 137 & V & por & R \\
\hline & & 138 & $\bar{L}$ & por & $R$ & 140 & $\mathrm{E}$ & por & $T$ & 141 & $Y$ & por & $\mathrm{F}$ & 143 & $S$ & por & A \\
\hline & & 145 & $F$ & por & C & 146 & A & por & $T$ & 147 & V & por & $\mathrm{L}$ & 148 & 1 & por & $\gamma$ \\
\hline & & 149 & 1 & por & $\mathrm{P}$ & 150 & $\mathrm{~s}$ & por & $\mathrm{E}$ & 151 & L & por & G & 152 & $\mathrm{H}$ & por & $\mathbf{R}$ \\
\hline & & 155 & $H$ & por & $M$ & 156 & A & por & $\bar{E}$ & 157 & $\bar{L}$ & por & V & 158 & $\mathrm{~F}$ & por & $\mathrm{H}$ \\
\hline & & 159 & $L$ & por & $\mathrm{S}$ & 162 & V & por & $\bar{K}$ & 163 & $R$ & por & $\mathrm{T}$ & 164 & A & por & G \\
\hline & & 165 & $\bar{V}$ & por & C & 166 & A & por & C & 177 & D & por & $E$ & 169 & $S$ & por & A \\
\hline & & 171 & $S$ & por & A & 173 & $\mathrm{R}$ & por & L & 174 & $\mathrm{~L}$ & por & $M$ & 175 & $\mathrm{~L}$ & por & $\mathrm{H}$ \\
\hline & & 176 & D & por & $R$ & 179 & A & por & $T$ & 180 & L & por & R & 182 & L & por & A \\
\hline & & 184 & P & por & C & 185 & G & por & D & 187 & $P$ & por & $G$ & 189 & A & por & G \\
\hline & & 191 & $T$ & por & A & 193 & $\mathrm{R}$ & por & $\mathrm{E}$ & 194 & L & por & R & 195 & L & por & I \\
\hline & & 196 & $\mathrm{R}$ & por & $\mathrm{K}$ & 197 & $Q$ & por & $\mathrm{K}$ & 198 & L & por & V & 199 & D & por & K \\
\hline & & 200 & A & por & P & 202 & V & por & $Y$ & 203 & $\mathrm{~F}$ & por & 1 & 204 & V & por & 1 \\
\hline & & 205 & A & por & 1 & 206 & Y & por & L & 207 & C & por & $\mathrm{F}$ & 208 & $\mathrm{~s}$ & por & A \\
\hline & & 210 & $E$ & por & $i$ & 212 & A & por & $S$ & 213 & $E$ & por & $Q$ & 214 & V & por & 1 \\
\hline & & 215 & $\bar{L}$ & por & $\mathrm{S}$ & 217 & A & por & $\mathrm{S}$ & 218 & $E$ & por & $Q$ & 219 & A & por & $P$ \\
\hline & & 220 & A & por & L & 221 & Q & por & G & 222 & A & por & $P$ & 223 & G & por & $\mathrm{S}$ \\
\hline & & 224 & L & por & S & 226 & G & por & $S$ & 228 & G & por & $\mathrm{S}$ & 229 & $\mathrm{H}$ & por & $\mathrm{F}$ \\
\hline & & 230 & $\bar{V}$ & por & $\bar{F}$ & 231 & W & por & $\mathrm{L}$ & 232 & $\mathrm{~L}$ & por & A & 233 & $\mathrm{~V}$ & por & i \\
\hline & & 234 & P & por & $\mathrm{S}$ & 235 & $\mathrm{~N}$ & por & G & 236 & $L$ & por & $\mathrm{S}$ & 240 & S & por & $\mathrm{F}$ \\
\hline & & 241 & $T$ & por & C & 242 & D & por & A & 243 & A & por & $F$ & 246 & A & por & S \\
\hline & & 247 & $T$ & por & G & 248 & $\mathrm{~F}$ & por & $\mathrm{L}$ & 249 & $P$ & por & R & 250 & V & por & P \\
\hline & & 252 & L & por & G & 253 & 1 & por & $\mathrm{V}$ & 255 & V & por & $Q$ & 256 & V & por & $\mathrm{s}$ \\
\hline & & 257 & $\bar{T}$ & por & $\mathrm{S}$ & 258 & $E$ & por & W & 281 & S & por & G & 283 & W & por & $\bar{R}$ \\
\hline & & 298 & V & por & A & 829 & $\mathrm{~S}$ & por & $T$ & 1114 & A & por & $\mathrm{T}$ & 1146 & $\mathrm{H}$ & por & Q \\
\hline & Rattus norvegicus & 5 & G & por & A & 6 & $M$ & por & V & 18 & $\mathrm{P}$ & por & L & 20 & A & por & $\mathrm{T}$ \\
\hline & & 24 & A & por & $\mathrm{F}$ & 28 & $\mathrm{P}$ & por & $\mathrm{s}$ & 32 & $\mathrm{~S}$ & por & $\mathrm{N}$ & 53 & $\mathrm{~L}$ & por & i \\
\hline & & 76 & G & por & D & 83 & 1 & por & $\mathrm{L}$ & 87 & 1 & por & V & 166 & $\mathrm{H}$ & por & $Y$ \\
\hline & & 198 & $\mathrm{~L}$ & por & V & 200 & $\mathrm{~F}$ & por & $\mathrm{L}$ & 244 & $\mathrm{~T}$ & por & A & 280 & w & por & $R$ \\
\hline & & 283 & $\mathrm{H}$ & por & $Y$ & 331 & Q & por & $\mathrm{R}$ & 352 & $E$ & por & D & 368 & A & por & $\mathrm{T}$ \\
\hline & & 422 & V & por & $\mathrm{L}$ & 423 & $\bar{A}$ & por & $T$ & 443 & $\mathrm{R}$ & por & $\mathrm{K}$ & 496 & V & por & I \\
\hline & & 512 & A & por & $\mathrm{S}$ & 564 & $\mathrm{R}$ & por & $\mathrm{K}$ & 568 & $S$ & por & $P$ & 756 & $\mathrm{~F}$ & por & L \\
\hline & & 844 & $\mathrm{~s}$ & por & $\mathrm{N}$ & 1044 & L & por & M & 1046 & $\mathrm{H}$ & por & $\mathbf{R}$ & 1104 & C & por & $\mathrm{R}$ \\
\hline & & 1107 & $T$ & por & $\mathrm{s}$ & 1109 & $\mathrm{R}$ & por & $P$ & 1113 & $Q$ & por & V & 1116 & E & por & V \\
\hline & & 1117 & $Q$ & por & P & 1120 & A & por & V & 1121 & P & por & A & 1122 & A & por & $T$ \\
\hline & & 1125 & $\mathrm{H}$ & por & $P$ & 1135 & A & por & $T$ & 1136 & $\mathrm{H}$ & por & $\mathrm{R}$ & 1143 & A & por & $T$ \\
\hline & & 1146 & P & por & $R$ & 1148 & L & por & $P$ & 1152 & A & por & $\mathrm{T}$ & 1155 & G & por & $\mathrm{s}$ \\
\hline & & 1156 & $S$ & por & P & 1159 & $\mathrm{~S}$ & por & 1 & 1161 & A & por & $\mathrm{T}$ & 1163 & G & por & $\mathrm{E}$ \\
\hline & & 1165 & L & por & P & 1166 & G & por & A & 1169 & G & por & V & 1183 & G & por & V \\
\hline & & 1190 & V & por & $E$ & 1199 & G & por & $R$ & 1200 & $P$ & por & S & & & & \\
\hline
\end{tabular}

Los cambios sombreados en verde representan cambios no conservados, los sombreados en amarillo son cambios menos conservados, los no sombreados son cambios conservados y los que se resaltan con un recuadro negro son cambios que se ubican en los dominios de unión a ligando S1 y/o S2. 
Tabla 5. Cambios Identificados en las Subunidades NR3A.

\begin{tabular}{|c|c|c|c|c|c|c|c|c|c|c|c|c|c|c|c|c|c|}
\hline \multirow{2}{*}{$\begin{array}{c}\text { Receptor } \\
\text { NR3A }\end{array}$} & \multirow{2}{*}{$\begin{array}{c}\text { Especie } \\
\text { Homo sapiens }\end{array}$} & \multirow{2}{*}{$\frac{\text { Posición }}{4}$} & \multicolumn{3}{|c|}{ Cambio } & \multirow{2}{*}{$\begin{array}{c}\text { Posición } \\
45\end{array}$} & \multicolumn{2}{|c|}{ Cambio } & \multicolumn{2}{|c|}{ Posición } & \multicolumn{3}{|c|}{ Cambio } & \multirow{2}{*}{$\begin{array}{c}\text { Posición } \\
596\end{array}$} & \multicolumn{3}{|c|}{ Cambio } \\
\hline & & & G & por & $\mathrm{S}$ & & G & por & D & 95 & $T$ & por & A & & S & por & $\mathrm{R}$ \\
\hline & & 664 & 1 & por & V & 859 & $S$ & por & A & 876 & V & por & M & 883 & $\mathrm{~K}$ & por & $\mathrm{R}$ \\
\hline & & 962 & V & por & 1 & 1015 & $\mathrm{R}$ & por & $Q$ & 1032 & S & por & L & 1033 & L & por & $\mathrm{R}$ \\
\hline & Pan troglodytes & 93 & A & por & $T$ & 112 & V & por & $M$ & 314 & $E$ & por & $\mathrm{K}$ & 596 & $S$ & por & $R$ \\
\hline & & 664 & 1 & por & v & 876 & v & por & $M$ & 883 & $\bar{K}$ & por & $\mathrm{R}$ & 1032 & S & por & $\mathrm{L}$ \\
\hline & Pongo pygmaeus & 34 & $T$ & por & A & 49 & G & por & A & 50 & A & por & $T$ & 62 & S & por & $P$ \\
\hline & & 66 & S & por & L & 323 & $\mathbf{P}$ & por & S & 423 & 1 & por & V & 507 & D & por & A \\
\hline & & 596 & S-R & por & $\mathrm{N}$ & 973 & $\mathrm{R}$ & por & $\mathrm{H}$ & 982 & $P$ & por & L & 1023 & P & por & L \\
\hline & Macaca mulata & 91 & A & por & $T$ & 92 & $\mathrm{R}$ & por & G & 361 & $\mathrm{H}$ & por & $\bar{Y}$ & 431 & V & por & 1 \\
\hline & & 957 & 1 & por & $\mathrm{L}$ & 1002 & 1 & por & $M$ & 1027 & $\mathrm{R}$ & por & $\mathrm{K}$ & & & & \\
\hline & Rattus norvegicus & 4 & G & por & $S$ & 43 & $\mathrm{P}$ & por & $Q$ & 46 & $\mathrm{~s}$ & por & G & 54 & $E$ & por & $\mathrm{D}$ \\
\hline & & 57 & $\mathrm{P}$ & por & $\mathrm{s}$ & 60 & $\mathrm{R}$ & por & $\mathrm{W}$ & 62 & $\mathrm{~S}$ & por & $\mathrm{P}$ & 75 & $T$ & por & A \\
\hline & & 87 & P & por & $L$ & 92 & $\mathrm{R}$ & por & G & 107 & D & por & $E$ & 138 & L & por & $M$ \\
\hline & & 188 & L & por & S & 195 & 1 & por & $\mathrm{L}$ & 258 & $Q$ & por & $E$ & 266 & $G$ & por & $\mathrm{E}$ \\
\hline & & 268 & 1 & por & V & 276 & $P$ & por & $S$ & 279 & $Q$ & por & $\mathrm{K}$ & 287 & 1 & por & V \\
\hline & & 289 & L & por & $M$ & 290 & $E$ & por & D & 291 & $\bar{S}$ & por & $\mathrm{N}$ & 293 & $\mathrm{~K}$ & por & $\mathrm{R}$ \\
\hline & & 299 & V & por & $M$ & 307 & $E$ & por & D & 311 & $\mathrm{R}$ & por & $Q$ & 315 & 1 & por & $M$ \\
\hline & & 316 & $T$ & por & $\mathrm{S}$ & 321 & V & por & $\mathrm{L}$ & 322 & M & por & $\mathrm{S}$ & 327 & $R$ & por & $\mathrm{H}$ \\
\hline & & 485 & $\mathrm{~N}$ & por & $S$ & 527 & $T$ & por & M & 530 & $S$ & por & $\mathrm{R}$ & 560 & K & por & Q \\
\hline & & 561 & 1 & por & L & 596 & $\mathrm{R}$ & por & $S$ & 600 & $\mathrm{H}$ & por & $\mathrm{N}$ & 677 & L & por & $M$ \\
\hline & & 685 & S & por & $\mathrm{N}$ & 706 & V & por & A & 804 & $E$ & por & $Q$ & 930 & $\mathrm{Y}$ & por & $\mathrm{H}$ \\
\hline & & 953 & L & por & $\mathrm{F}$ & 957 & 1 & por & $\mathrm{L}$ & 967 & Q & por & $P$ & 968 & $\mathrm{H}$ & por & $\mathrm{R}$ \\
\hline & & 969 & $F$ & por & S & 1000 & V & por & $\mathrm{L}$ & 1003 & $\mathrm{R}$ & por & $Q$ & 1006 & $T$ & por & $M$ \\
\hline & & 1018 & $\mathrm{R}$ & por & Q & 1024 & $S$ & por & $N$ & 1034 & 1 & por & $T$ & 1035 & R & por & Q \\
\hline & & 1036 & 1 & por & A & 1044 & P & por & 0 & 1065 & $\mathrm{~S}$ & por & $T$ & 1067 & $\mathrm{~N}$ & por & $\mathrm{s}$ \\
\hline & & 1070 & M & por & 1 & & & & & & & & & & & & \\
\hline
\end{tabular}

Los cambios sombreados en verde representan cambios no conservados, los sombreados en amarillo son cambios menos conservados, los no sombreados son cambios conservados y los que se resaltan con un recuadro negro son cambios que se ubican en los dominios de unión a ligando S1 y/o S2.

\section{Predicción de Estructura Secundaria}

Esta predicción se realizó con el propósito de reconocer si las sustituciones identificadas en la región C-terminal influían en cambios de estructura. En la subunidad Glur5 se observa que las especies Homo sapiens y Pan troglodytes presentan la misma estructura en su región C-terminal (datos no mostrados), mientras que para las especies Pongo pygmaeus, Macaca mulata y Rattus norvegicus se observan las siguientes diferencias: la especie Pongo pygmaeus presenta una secuencia más larga (915 aminoácidos) y las diferencias se presentan a partir del aminoácido 848 con la presencia adicional de una hoja $\beta$, dos hélices $\alpha$ y regiones de baja estructuración, en la especie Macaca mulata la secuencia es más corta ( 873 aminoácidos), su estructura es semejante a la de las otras especies sin embargo no coincide en la posición de los aminoácidos y finaliza con una región de baja estructuración desde el aminoácido 838 .

En la subunidad NR2A se presentan pequeños cambios estructurales a partir del aminoácido 845, la especie Homo sapiens presenta una hélice $\alpha$ entre los residuos 980-990 y la especie Macaca mulata presenta dos hélices $\alpha$ entre los residuos 1263-1273 y 1312-1319 y finaliza su extremo C-terminal con una baja estructuración en comparación a las otras especies donde se observan tres hélices $\alpha$ (datos no mostrados).
En la subunidad NR2C la región C-terminal está caracterizada por presentar una baja estructuración a partir del aminoácido 847 para las especies Homo sapiens y Macaca mulata, mientras que en las demás especies aunque predomina una baja estructuración o coil se presentan cuatro hélices $\alpha$ en Pan troglodytes $y$ dos hélices $\alpha$ en Pongo pygmaeus (datos no mostrados).

En la subunidad NR3A se presentan pequeños cambios de estructura entre los aminoácidos 944-958 en la especie Homo sapiens se observa una hélice $\alpha$ y dos hojas $\beta$, en Pan troglodytes una hélice $\alpha$ y una hoja $\beta$ y en Pongo pygmaeus y Macaca mulata una hélice $\alpha$, y entre los residuos 1023-1035 Homo sapiens presenta dos hélices $\alpha$, Pongo pygmaeus una hélice $\alpha$ y una hoja $\beta$ y Pan troglodytes y Macaca mulata una hélice $\alpha$ (datos no mostrados).

\section{Estimación de sitios de fosforilación}

Se identificó la presencia de nuevos sitios de fosforilación y la ausencia de otros en las secuencias de la subunidad GluR5 para las especies Homo sapiens y Pan troglodytes, así como en Macaca mulata para la subunidad NR2A y en Pan troglodytes para la subunidad NR2C (Tabla 6). Por lo tanto sería interesante comprobar experimentalmente de qué forma los cambios en estructura y sitios de 
fosforilación alteran la afinidad por las proteínas que se unen a motivos en el dominio C-terminal de las subunidades mencionadas.

\section{Estimación filogenética}

La reconstrucción filogenética consenso se llevó a cabo empleando el modelo de evolución JTT $+\mathrm{I}+\mathrm{G}+\mathrm{F}$ que se basa en el intercambio de aminoácidos estimado a partir de matrices de bases de datos de las alineaciones de proteínas, que incorporan una frecuencia promedio de aminoácidos. El árbol obtenido (Figura 1) refleja en su mayoría las topologías o formas de las ramas observadas en las reconstrucciones por especie, por subunidad y por receptor (datos no mostrados). Se puede observar que las subunidades se relacionan en grupos o linajes de acuerdo al receptor, a excepción de las subunidades NR2 donde se destaca que la subunidad más cercana al nodo ancestral de los iGLURs es NR2D.

La subunidad NR2D se encuentra relacionada con los nodos que originan a las subunidades NR2C y NR2A y B, a su vez las subunidades NR2A y B aparecen más estrechamente relacionadas con los nodos que originan a las subunidades NR3 y NR1, finalmente las subunidades NR1 comparten una relación estrecha con el nodo que origina a las subunidades AMPA y Kainato (Figura 1).

\section{Discusión}

Los alineamientos múltiples permitieron identificar los cambios que se presentan en las diferentes subunidades de los receptores NMDA, AMPA y Kainato, dentro de los que se destacan las sustituciones que involucran los dominios de unión aligando S1 y/o S2, y que fueron identificados teniendo en cuenta los reportes de estudios de cristalografía con residuos de unión al agonista en las subunidades GluR2, GluR5, GluR6, NR2A y NR1, y por comparación para los demás iGluRs (17), estos resultados sugieren variaciones en la interacción con el glutamato particularmente en la subunidad NR3A, dada la variación de los residuos en la posición 596 en las cuatro especies de primates, por lo que sería importante comprobar a través de estudios de dinámica molecular el papel que juega este residuo en el dominio de unión $\mathrm{S} 1$.

El dominio C-terminal de los iGluRs sirve como plataforma de importantes modificaciones postraduccionales, representa un buen dominio de anclaje para interacciones proteína-proteína con una variedad de proteínas que están envueltas en el direccionamiento, tráfico y anclaje de estos receptores (18). Adicionalmente esta región presenta importantes sitios de fosforilación para serina, treonina y tirosina quinasas como es el caso de la proteína quinasa $C$, proteína quinasa $A$, entre otras (19). Lo anterior resulta importante teniendo en cuenta que las subunidades GLUR5, NR2A, NR2C y NR3A se destacaron por presentar una mayor proporción de sustituciones en la región C-terminal, que pueden estar influyendo en los cambios de estructura secundaria observados a través de la predicción, adicionalmente el reconocimiento de variaciones en los sitios de fosforilación en el C-terminal de las subunidades GLUR5, NR2A y NR2C plantean la posibilidad de que las interacciones con proteínas que se unen a dominios ubicados en esta región puedan estar variando. Por lo tanto sería importante complementar es-

Tabla 6. Sitios de fosforilación que hacen parte de los cambios

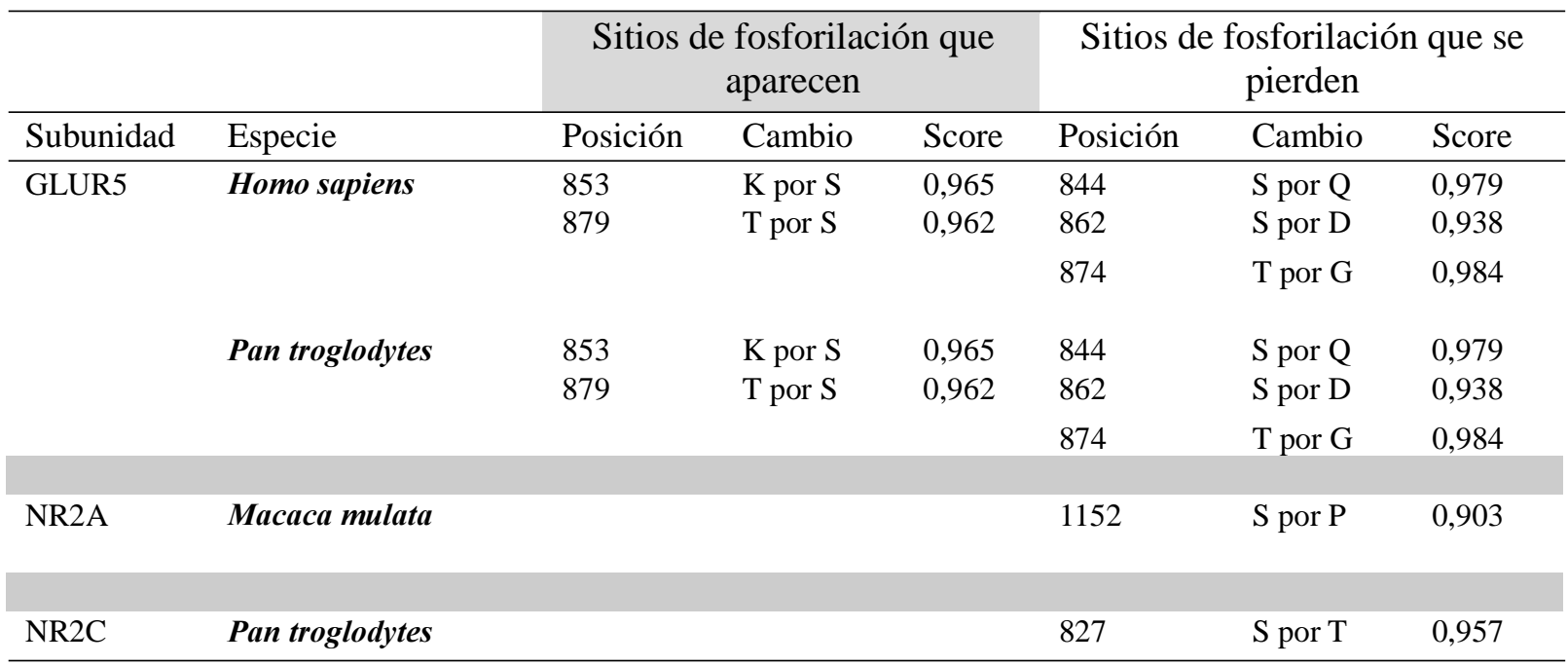




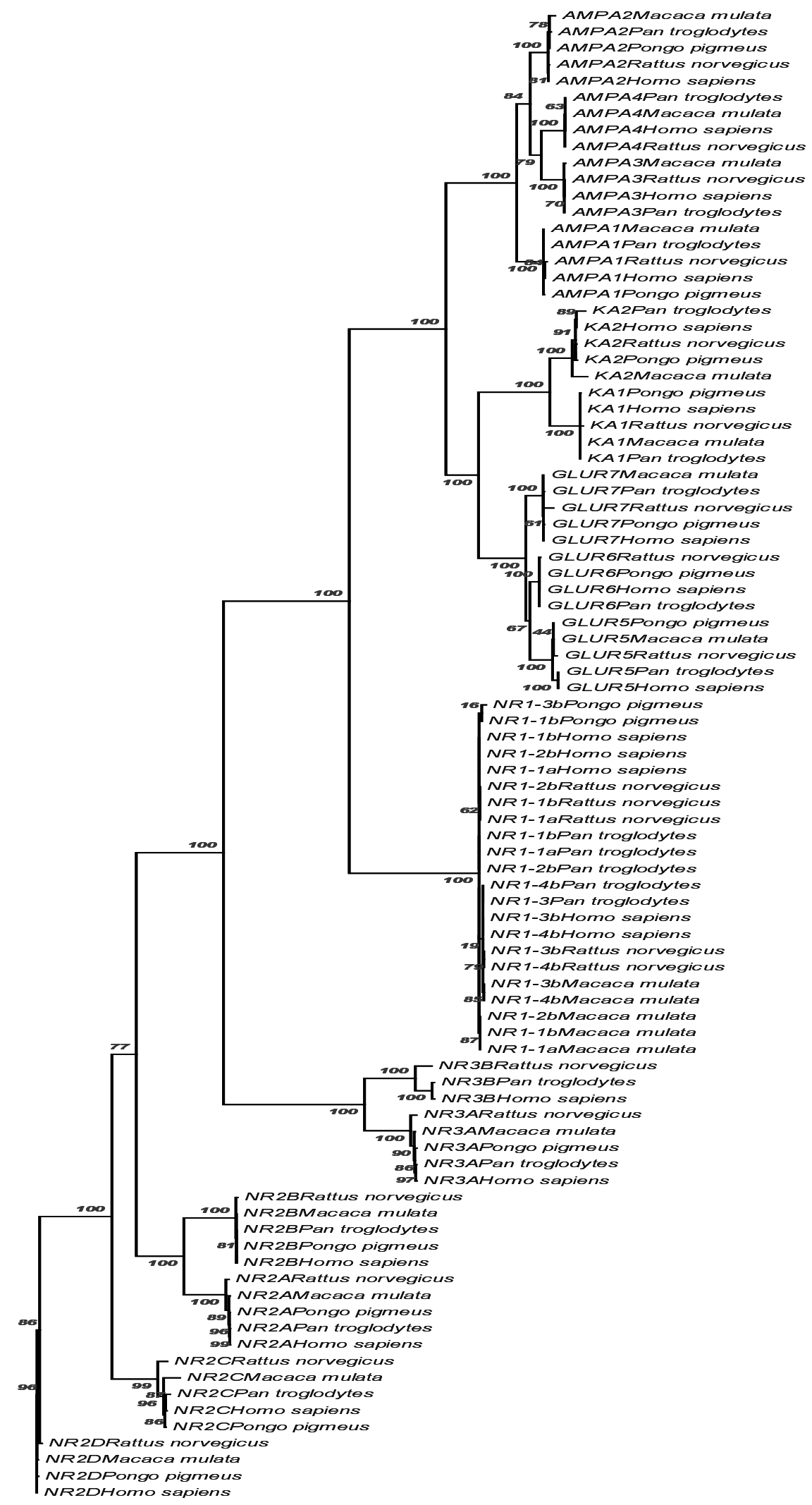

Figura 1. Árbol Filogenético de los receptores ionotrópicos de Glutamato para las especies Homo sapiens, Pan troglodytes, Pongo pygmaeus, Macaca mulata y Rattus norvegicus. El método de reconstrucción filogenética empleado fue Máxima Verosimilitud, el modelo de evolución seleccionado fue JTT+I+G+F y un Bootstrap de 100. 
tos resultados con estudios de predicción de estructura terciaria.

Las subunidades de los receptores Kainato interactúan con numerosas proteínas que afectan su función y distribución intracelular, investigaciones en esta área se han centrado en la influencia de las proteínas con dominios PDZ, como es el caso de las proteínas PSD-95, GRIP y PICK1 que interactúan en diferentes grados con los motivos que se ubican en el C-terminal de las subunidades de Kainato e involucran aminoácidos como serina y treonina, lo que sugiere una interacción proteína-proteína por fosforilación (17).

Para las subunidades del receptor NMDA también existe una variedad de proteínas que actúan directamente con el dominio C-terminal como es el caso de las proteínas MAGUK (PSD-95, PSD-93, SAP97 y SAP102), estas proteínas presentan tres dominios PDZ que se unen a los dominios PDZ de las subunidades NMDA y forma un complejo molecular que se puede asociar a otras proteínas alrededor de la densidad post-sináptica (PDS), favoreciendo la unión de los receptores NMDA a otros receptores de glutamato o canales iónicos (20-22).

La identificación por predicción de sitios de fosforilación que aparecen o se pierden en la región C-terminal, a través de los cambios que presentan las subunidades GluR5 en las especies Homo sapiens y Pan troglodytes, NR2A en la especie Macaca mulata y NR2C en la especie Pan troglodytes, proponen la existencia de variaciones en la interacción de estas subunidades en las especies correspondientes con proteínas quinasas y con proteínas con dominios PDZ que se unen a los dominios PDZ en la región C-terminal de los iGluRs y puedan estar alterando la conformación de complejos moleculares alrededor de la densidad postsináptica (PDS), por lo tanto se hace necesario comprobar de forma experimental como afecta o favorece la interacción con proteínas con dominios PDZ como PSD-95, PSD-93 y proteínas quinasas la presencia de nuevos sitios de fosforilación y la ausencia de otros, en las secuencias de la subunidad GluR5, NR2A y NR2C.

La reconstrucción filogenética consenso sugiere que las subunidades se relacionan en linajes de acuerdo al receptor, excepto para el receptor NMDA, en este caso las subunidades NR1 están más estrechamente relacionadas con el nodo que origina las subunidades de los receptores AMPA y Kainato, de igual forma se relacionan por una rama ancestral con las subunidades NR3, las cuales a su vez se relacionan con el nodo que origina a las subunidade4s NR2, dentro de las cuales las subunidades NR2D se destacan por ser las más cercanas al nodo ances- tral de los iGluRs. Lo anterior sugiere que los cambios que evolutivamente se presentaron en las subunidades NR2 dada su cercanía al nodo ancestral dieron origen a las demás subunidades de los receptores ionotrópicos de glutamato.

La topología observada se relaciona con los resultados obtenidos en los análisis de parsimonia realizado para Genes iGLURs en animales y genes GLR en plantas como Arabidopsis thaliana (9), donde se mostró que los receptores AMPA y Kainato están más estrechamente relacionados entre sí, comparados con los receptores NMDA y la cercanía de las subunidades NMDA al ancestro común sugiriendo que la divergencia que se presentó en estas subunidades dio origen a los demás iGLURs.

También es de resaltar que en las subunidades NR2, se presenta una relación más estrecha entre NR2A y NR2B y entre NR2C y NR2D, estos resultados son acordes con los propuestos en estudios previos, donde se sugiere que evolutivamente se produjo una duplicación que dio origen a los cuatro parálogos de NR2 (10).

\section{Conclusiones}

La identificación por predicción de sitios de fosforilación que aparecen o se pierden en la región C-terminal a través de los cambios que se presentan las subunidades GluR5, NR2A y NR2C sugieren la existencia de variaciones en la interacción de estas subunidades en las especies correspondientes con proteínas quinasas y con proteínas con dominios PDZ que están involucradas en los procesos de direccionamiento, tráfico y anclaje de los receptores en las posiciones intracelulares. La estimación filogenética general propone que los cambios que evolutivamente se presentaron en las subunidades NMDA y posiblemente en la subunidad NR2D dieron origen a las demás subunidades de los receptores ionotrópicos de glutamato

\section{Agradecimientos}

A los integrantes del grupo de investigación de Bioquímica Molecular Computacional, Estructural y Bioinformática de la Pontificia Universidad Javeriana y en especial a las profesoras Janneth Gonzalez y Sonia Luz Albarracín por su oportuna orientación.

\section{Financiación}

Este trabajo se realizó gracias a la plataforma computacional disponible en el laboratorio del grupo de inves- 
tigación de Bioquímica Molecular Computacional, Estructural y Bioinformática de la Pontificia Universidad Javeriana.

\section{Conflictos de intereses}

Los autores declaran que no existen conflictos de intereses en relación a este trabajo.

\section{Referencias}

1. Boyd R, Silk J. Cómo Evolucionaron Los Humanos. En: Ariel, S.A. Barcelona, España. 2001; 11-35, 132153.

2. Moore BR. The Evolution of Learning. Biological Reviews of the Cambridge Philosophical Society. 2004; 9: 301-335.

3. Dorus S, Vallender EJ, Patrick D, Evans P, Anderson J, Gilbert S, Mahowald M, Wyckoff G, Malcom C, Lahn B. Accelerated Evolution of Nervous System Genes in the Origin of Homo sapiens. Cell. 2004; 119: $1027-1040$.

4. Nakanishi S. Molecular diversity of glutamate receptors and implications for brain function. Science. 1992; 258: 597-603.

5. Monaghan D, Bridges R, Cotman, C. The Excitatory Amino Acid Receptors:Their Classes, Pharmacology, and Distinct Properties in the Function of the Central Nervous System. Annual Review of Pharmacology and Toxicology. 1989; 29: 365-402.

6. Maren S, Baudry M. Properties and Mechanisms of Long-Term Synaptic Plasticity in The Mammalian Brain Relationships to Learning and Memory. Neurobiology of Learning and Memory. 1995; 6: 1-18.

7. Mayer M, Armstrong N. Structure and Function of Glutamates Receptor Ion Channels. Annual Review of Physiology. 2004; 66: 161-181.

8. Dingledine R, Borges K, Bowie D, Traynelis, S. The Glutamate Receptor Ion Channels. The American Society for Pharmacology and Experimental Therapeutics. 1999; 51: 7-61.

9. Chiu J, Rob Desalle R, Lam H, Meisel L, Coruzzi G. Molecular Evolution of Glutamate Receptors: A Primitive Signaling Mechanism that Existed Befote Plants and Animals Diverged. Molecular Biology Evolution. 1999; 16: 826-838.
10. Ryan T, Emes R, Grant G, Komiyama N. Evolution of NMDA Receptor Cytoplasmic Interaction Domains: Implications for Organisation of Synaptic Signallin Complexes. BMC Neuroscience. 2008; 9: 1-14.

11. Jeanmougin F, Thompson J, Gouy M, Higgins D, Gibson T. Multiple sequence alignment with Clustal X. Trends in Biochemical Sciences.1998; 23: 403-405.

12. McGuffin L, Bryson K, Jones, D. The PSIPRED protein structure prediction server. Bioinformatics. 2000; 16: 404-405.

13. Blom N, Gammeltoft S, Brunak S. Sequence and Structure-Based Prediction of Eukaryotic Protein Phosphorylation Sites. Journal Molecular Biology. 1999; 294: 1351-1362.

14. Abascal F, Zardoya R, Posada D. ProtTest: selection of best-fit models of protein evolution. Bioinformatics. 2005; 21: 2104-2105.

15. Chen S, Su S, Lo C, Chen K, Huang T, Kuo B, Lin C. PALM: A Paralleled and Integrated Framework for Phylogenetic Inference with Automatic Likelihood Model Selectors. PLOS ONE. 2009; 14: 1-6.

16. Guindon S, Gascuel O. A Simple, Fast, and Accurate Algorithm to Estimate Large Phylogenies by Maximum Likelihood. Systematic Biology. 2003; 52: 696704.

17. Gereau R, Swanson G. The Glutamate Receptor. En: Human Press. 2008; 68-69, 130-131, 262-265.

18. Scannevin R, Huganir R. Postsynaptic organization and regulation of excitatory synapses. Nature Reviews Neuroscience. 2000; 1: 133-141.

19. MacDonald J, Kotecha S, Lu W, Jackson M. Convergence of PKC-Dependent Kinase Signal Cascades on NMDA Receptors. Current Drug Targets. 2001; 2: 299-312.

20. Cull-Candy S, Brickley S, Farrant M. NMDA Receptor Subunits: Diversity, Development and Disease. Current Opinion in Neurobiology. 2001; 11: 327-335.

21. Lim I, Merrill M, Chen Y, Hell J. Disruption of the NMDA Receptor-PSD-95 Interaction in Hippocampal Neurons with no Obvious Physiological Short-Term Effect. Neuropharmacology. 2003; 45: 738-754.

22. Kim E, Sheng M. PDZ Domain Proteins of Synapses. Nature Reviews Neuroscience. 2004; 5: 771-781. 